\title{
Une vision pour la pharmacie hospitalière au Canada
}

\author{
par Neil J MacKinnon
}

Faute de vision, le peuple vit sans frein. — Les Proverbes 29:18 (version de La Bible de Jérusalem)

Cón omme beaucoup d'entre vous le savent, chacun des trois délégués présidentiels de la SCPH est responsable d'une fonction différente (liaison externe, liaison interne ou vision) pour la durée de son mandat de trois ans. À titre d'agent de liaison pour la vision de la SCPH, je n'ai pas le mandat de développer personnellement une vision pour la pharmacie hospitalière au Canada (et Dieu merci), mais beaucoup de mes tâches ont trait à la planification, à la surveillance et à l'évaluation de l'atteinte des objectifs de la Société.

Alors, que veut-on dire par le mot "vision »? Comme le roi Salomon l'a dit il y a environ 3000 ans dans le proverbe cité plus haut, une vision peut être très influente et puissante. D'ailleurs, le Petit Robert donne plusieurs définitions du mot "vision ", notamment une " action de voir, de se représenter en esprit » loin du type de vision auquel le roi Salomon faisait référence ou que l'agent de liaison pour la vision de la SCPH appréhende. Une définition plus pertinente serait " une idée ou un but vers lequel on tend ".

La Vision 2011 de la SCPH, adoptée en 2007, est de devenir une Société dynamique, la voix influente de la pharmacie hospitalière, une inspiration pour l'excellence de la pratique et une source de leadership et de perfectionnement professionnel. La SCPH a mis en place plusieurs mesures du rendement qui font l'objet d'un suivi périodique pour ainsi aider la SCPH à concrétiser cette vision. Plus particulièrement, un nouveau plan de développement stratégique, qui est en train d'être élaboré au moment où je rédige cet article, contribuera à donner une orientation à la SCPH durant les trois prochaines années.

Quelle est la vision de la SCPH pour ce qui est de la pratique de la pharmacie hospitalière au Canada? L'une des façons les plus concrètes par laquelle la SCPH exprime sa vision est le projet SCPH 2015, un programme visant l'excellence dans la pratique pharmaceutique pour les pharmaciens œuvrant dans les établissements de santé. Lobjet général de ce programme est d'accroître l'utilisation efficace, scientifique et sûre des médicaments et de contribuer significativement à la santé publique, pour concourir ainsi à la bonne santé des Canadiens.
La réussite sera au rendez-vous lorsque seront atteints les six buts assortis des 36 objectifs mesurables.

La SCPH a aussi adhéré à la vision énoncée dans le Plan directeur pour la pharmacie : "Procurer aux Canadiens un traitement médicamenteux aux résultats optimaux par des soins axés sur le patient » (visitez www.pharmacists.ca/content/about_cpha/ whats_happening/cpha_in_action/pdf/BlueprintVision.pdf). La SCPH continue de collaborer activement avec d'autres organismes de pharmacie pour concrétiser cette vision dans notre profession. Par exemple, cet automne, la SCPH participera au projet de modèle de pratique de la pharmacie de l'American Society of Health-System Pharmacists, pour contribuer à forger une nouvelle vision spécifique de la pratique de la pharmacie hospitalière.

On remarquera aussi que le mot "vision " recouvre une autre définition, à savoir " représentation imaginaire ", un peu comme rêver de quelque chose... Ce lien entre la vision et le rêve a été bien décrit par le $\mathrm{D}^{\mathrm{r}}$ Charles $\mathrm{D}$. Hepler dans son discours d'acceptation du prix H. A. K. Whitney 2010 (prononcé dans le cadre du congrès et de l'exposition de l'ASHP au mois de juin 2010; voir Am J Health Syst Pharm 2010;67[16]:13191325) dans lequel il dit que [traduction libre] " Le rêve de la pharmacie en tant que profession clinique est différé depuis trop longtemps » et "Il n'y aura jamais de meilleur moment pour nous de transformer notre rêve différé en une vision réalisée. "

Notre profession a fait d'incroyables progrès au cours des dernières décennies, avec la montée de la pharmacie clinique et des soins pharmaceutiques. Mais il reste encore beaucoup de travail à faire. Les pharmaciens ne prodiguent pas des soins pharmaceutiques à tous les patients des hôpitaux de notre pays, et il y a des patients pour qui le système de distribution des médicaments n'est ni sûr, ni efficace. Le défi que je vous lance est de faire passer le mot "vision " sous ses différentes facettes dans votre domaine de pratique alors que vous rêvez de prodiguer de nouveaux services et redoublez d'effort pour atteindre les cibles du projet SCPH 2015.

[Traduction par l'éditeur]

Neil J MacKinnon, , B. Sc. (Pharm.), M. Sc. (Pharm.), Ph. D., FCSHP, est président et agent de liaison pour la vision de la SCPH. 